\title{
Anterior decompression through transoral axis slide and rotation osteotomy for salvage of failed posterior occipitocervical fusion: a novel technique note
}

\author{
Aimin Wu", Haiming Jin", Haicheng Dou, Xiangxiang Pan, Sunren Sheng, Chongan Huang, Xiangyang Wang \\ Division of Spine Surgery, Department of Orthopaedics, The Second Affiliated Hospital and Yuying Children's Hospital of Wenzhou Medical \\ University, Zhejiang Spine Surgery Center, Key Laboratory of Orthopaedics of Zhejiang Province, Wenzhou 325027, China \\ \#These authors contributed equally to this work. \\ Correspondence to: Xiangyang Wang, MD, PhD. Division of Spine Surgery, Department of Orthopaedics, The Second Affiliated Hospital and Yuying \\ Children's Hospital of Wenzhou Medical University, Zhejiang Spine Surgery Center, Key Laboratory of Orthopaedics of Zhejiang Province, 109\# \\ XueYuan Western Road, Wenzhou 325027, China. Email: xiangyangwang@126.com.
}

\begin{abstract}
Atlantoaxial dislocation could be caused by odontoid fractures or Os odontoideum. The previous surgical techniques in treatment of atlantoaxial dislocation were based on arch remove decompression or anterior atlantoaxial release and atlantoaxial (occipital-cervical) screw fixation-based reduction and fusion. However, for some clinical situations, all of above techniques cannot be applied. In this study, a patient with atlantoaxial dislocation caused by Os odontoideum treated by posterior occipitocervical fusion 20 years ago and failed. We design a novel anterior decompression through transoral axis slide and rotation osteotomy for salvage of this failed posterior occipitocervical fusion case. The C2 body and odontoid process was ventrally slide and rotation at good position after operation as well as the position of plate and screws, the spinal canal was increased significantly after operation too. We suggest this anterior decompression through transoral " $\mathrm{C} 2$ slide and rotation" technique is good choice for salvage of failed posterior occipitocervical fusion and some irreducible atlantoaxial dislocation because of the anterior bony fusion, it could direct decompress the spinal cord anteriorly, avoid the odontoid resection, and is feasible and safe technique.
\end{abstract}

Keywords: Atlantoaxial dislocation; anterior decompression; transoral approach; axis slide and rotation; occipitocervical fusion; technique note; upper cervical osteotomy

Submitted Feb 03, 2020. Accepted for publication Feb 06, 2020.

doi: $10.21037 /$ atm.2020.02.39

View this article at: http://dx.doi.org/10.21037/atm.2020.02.39

\section{Introduction}

The complex of occipitocervical anatomy makes the high risk of surgery in this region (1-4). The upper cervical injuries might lead paraplegia or death (5-7). Atlantoaxial dislocation could be caused by odontoid fractures or Os odontoideum (8-10). The posterior decompression, reduction and fusion techniques are the mainly treatments for atlantoaxial dislocation $(11,12)$. For irreducible atlantoaxial dislocation, some authors try the anterior odontoid osteotomy and posterior fusion $(13,14)$, or anterior atlantoaxial release and posterior fusion (15-17).

To summary the previous literatures, the techniques were based on arch remove decompression or/and atlantoaxial (occipital-cervical) screw fixation-based reduction and fusion. However, for some unique situations, all of above techniques cannot be applied. A patient with atlantoaxial dislocation caused by Os odontoideum treated by posterior occipitocervical fusion 20 years ago and failed. We design a novel anterior decompression through transoral axis slide and rotation osteotomy for salvage of this failed posterior occipitocervical fusion case. 


\section{Patient information}

A 56-year-old female patient was diagnosed as Os odontoideum and atlantoaxial dislocation at 20 years ago, at that time, she was treated with posterior wire fixation and bone graft fusion by another surgeon. The patient said she had good clinical outcomes at that time, and didn't back for follow up. One month before this hospital admission, she had a mild fall down, and felt the progressive weakness of the four limbs. She went to her local hospital, and the local hospital refer her to our hospital. When she admitted in our hospital, she couldn't walk by herself. The physical examination found that the muscle strength grades of left upper limb, right upper limb, left lower limb, right lower limb was 2, 3, 1, 3, respectively. Hoffmann sign: L (+), R (+); Babinski sign: $\mathrm{L}(+), \mathrm{R}(+)$.

The preoperative anteroposterior open mouth and lateral radiographical images (Figure $1 A, B$ ) found the atlantoaxial dislocation and posterior wire fixation. The preoperative CT images (Figure 1C,D) gave us more details of the posterior bony fusion, showed that the bony fusion achieved from the occipital to $\mathrm{C} 4$, but the $\mathrm{C} 1 / 2$ fusion is suspected not very stable, one metal wire was transversal walking behind the spinal cord and compress the spinal cord posteriorly, while the upper-posterior of the odontoid process compress the spinal cord anteriorly, very narrow width space for the spinal cord, dura mater and cerebrospinal fluid. The preoperative MR images (Figure $1 E, F$ ) also showed the narrow space between the transversal walking wire and upper-posterior of the odontoid process.

\section{Surgical technique}

The patient was placed supine on the operating table with fiber-optic nasotracheal intubation, the head and neck stabilized and traction of $3 \mathrm{~kg}$ applied to Gardner-Wells calipers. Neurophysiologic monitoring involving SSEPs (somatosensory-evoked potentials) and MEPs (motorevoked potentials) were used to monitor the potential neurologic deficit. The skin was prepared and draped with sterile fashion; the oropharynx was prepared with povidoneiodine solution.

The transoral retractor was inserted with the help of tongue blade, the uvula and soft palate was pulled out of the operative field. The wound incision was made at the wall of the posterior pharynx to expose the region from $\mathrm{C} 1$ to $\mathrm{C} 3$. First, the routine discectomy was carried out in the level of $\mathrm{C} 2 / 3$, then, we used the ultrasonic scalpel to perform the bilateral osteotomy of the $\mathrm{C} 2$ body (Figure $2 A$ ), the region of bilateral osteotomy from the internal side of the bilateral uncovertebral joint to the $1 / 3$ internal point of the C1 lateral mass, small inferior-internal partial of the C1 lateral mass was also removed to obtain enough space for odontoid process and the removed C2 body ventrally moved. After the bilateral osteotomy of the $\mathrm{C} 2$ body, the middle float C2 body was hold by Cook forceps in case of it backward move to increase the spinal cord compression, and the soft tissue connection and scars were removed by the 1-2 mm Kerrison rongeurs or nerve dissector. We also used the high-speed burr to remove the antero-inferior raised portion of $\mathrm{C} 2$ body and anterior raised portion of $\mathrm{C} 1$ arch as well as some osteophytes, to make the front of $\mathrm{C} 1$, $\mathrm{C} 2$ and $\mathrm{C} 3$ to be a smooth plane (Figure $2 A$ ).

Then, anterior cervical titanium alloy plate was placed at the front of $\mathrm{C} 1-\mathrm{C} 3$. In order to let the $\mathrm{C} 2$ body conform to the anterior cervical titanium alloy plate, the lower part of the $\mathrm{C} 2$ body was ventrally slide to the anterior plate, while the upper part of $\mathrm{C} 2$ body and odontoid process was ventrally rotated with the antero-inferior edge of $\mathrm{C} 2$ as the fulcrum (Figure 2B), therefore, we named this technique as anterior decompression through transoral " $\mathrm{C} 2$ slide and rotation" technique. Finally, the bilateral screws for each vertebral body were introduced to fixate the plate (Figure 2C), at this time, to avoid the $\mathrm{C} 2$ body backward move, the C2 body should be held by Cook forceps, and the screw trajectories were made by high-speed burr. The autogenous cancellous graft was placed at bilateral lateral atlantoaxial joint space and bilateral osteotomized gap. The posterior pharynx was closed by interrupted sutures.

\section{Results}

The postoperative anteroposterior open mouth and lateral radiographical images showed the plate and screws placed at good position (Figure $3 A, B$ ), the $\mathrm{C} 2$ body and odontoid process was ventrally slide and rotation at good position too, the spinal canal was increased significantly after operation (Figure 3C). The muscle strength grades of left upper limb, right upper limb, left lower limb, right lower limb was recovered to $4,5,4,5$ after operation, respectively.

\section{Discussion}

The compression of the upper cervical spinal will induce the paraplegia or death $(5,6)$, enlarge the spinal canal and liberate the compression is the key therapy to stop 

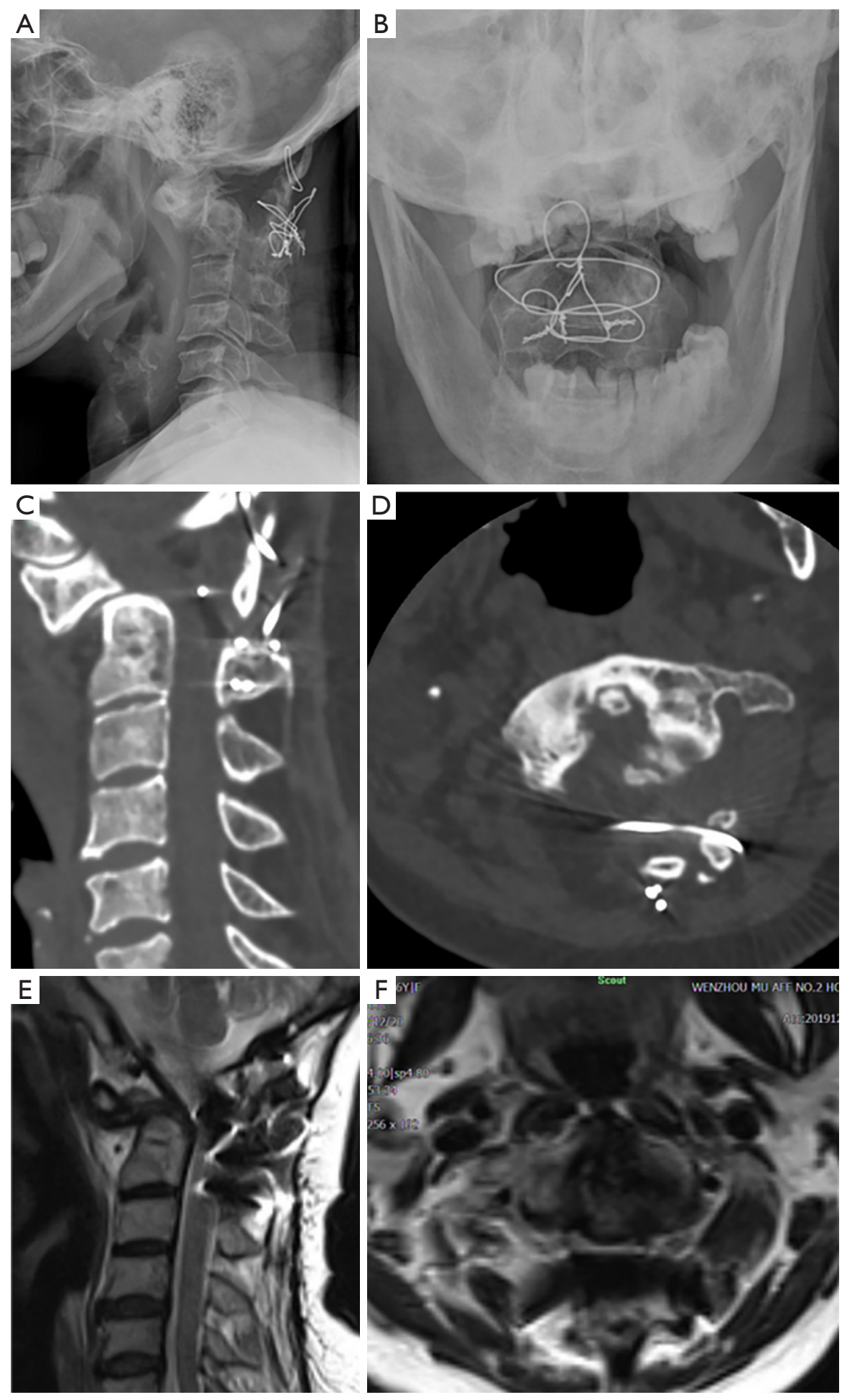

Figure 1 A 56-year-old female patient was diagnosed as Os odontoideum and atlantoaxial dislocation at 20 years ago. The pre-operative $\mathrm{X}$-ray of this hospital admission found that the atlantoaxial dislocation and posterior wire fixation (A,B); The preoperative CT showed that one metal wire was transversal walking at the front of $\mathrm{C} 1$ posterior arch, compress the spinal cord posteriorly, while the upper-posterior of the odontoid process compress the spinal cord anteriorly (C,D); the preoperative MRI also showed the narrow space between the transversal walking wire and upper-posterior of the odontoid process $(\mathrm{E}, \mathrm{F})$. 

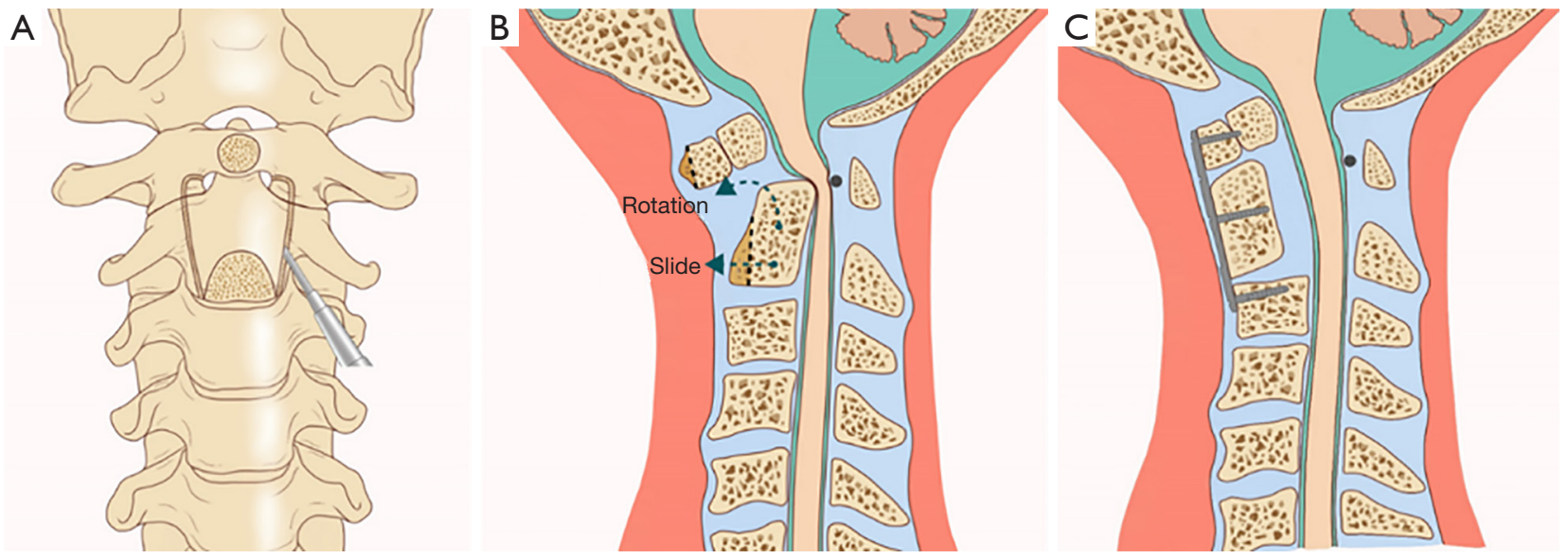

Figure 2 The schematic graph of our novel anterior transoral axis sliding and rotation osteotomy. (A) The antero-inferior raised portion of $\mathrm{C} 2$ body and anterior raised portion of $\mathrm{C} 1$ arch was removed by the high-speed burr, the bilateral osteotomy (from internal side of the uncovertebral joint to the $1 / 3$ internal point of the $\mathrm{C} 1$ lateral mass) was performed by the ultrasonic scalpel; (B) the lower part of the $\mathrm{C} 2$ body was ventrally slide, while the upper part of $\mathrm{C} 2$ body and odontoid process was ventrally rotated with the antero-inferior edge of $\mathrm{C} 2$ as the fulcrum, the shadow part in the schematic graph was removed part by high-speed burr to conform to the anterior cervical plate; (C) the bilateral screws for each vertebral body were introduced to fixate the plate on C1-C3, the spinal canal was increased significantly at this time.
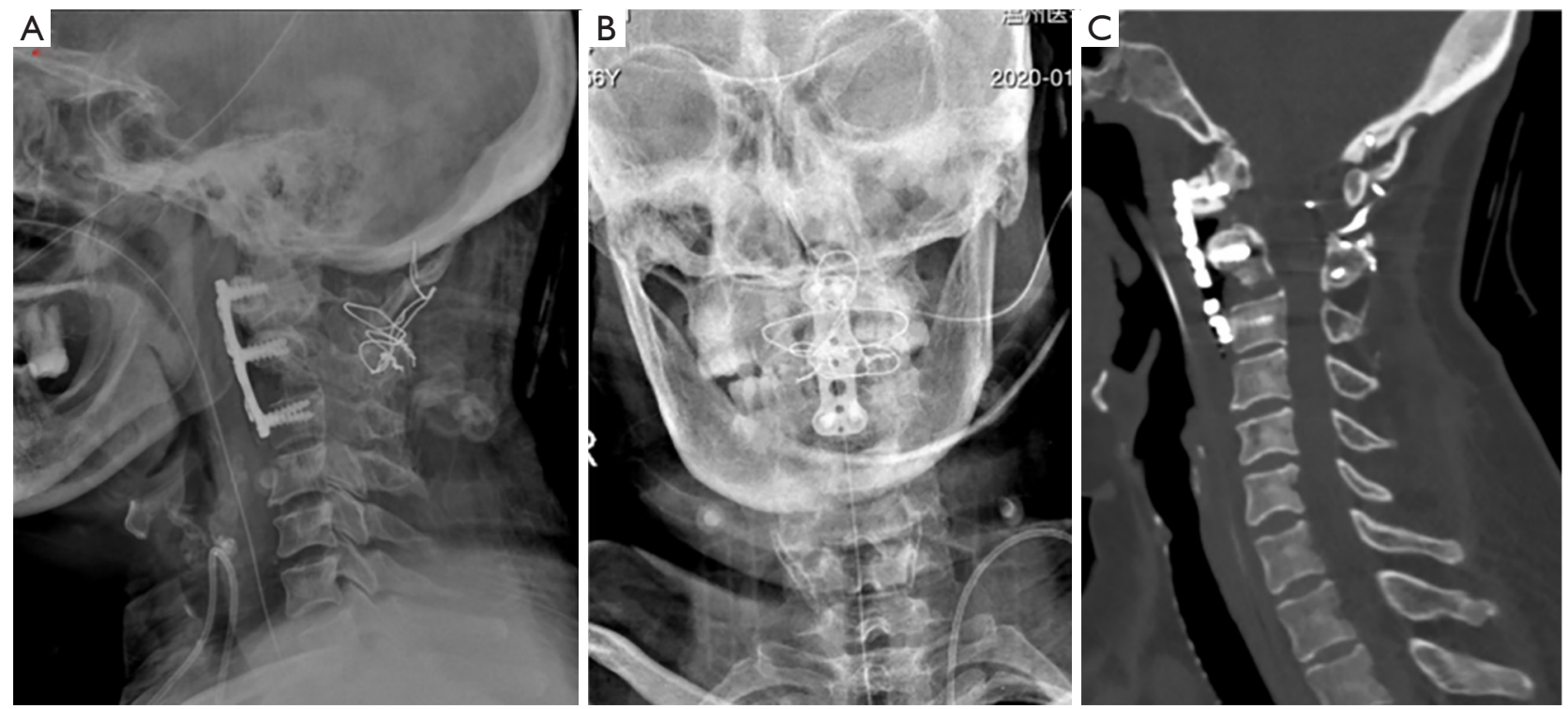

Figure 3 The postoperative images of this case. (A,B) The postoperative anteroposterior open mouth and lateral radiographical images showed that the plate and screws placed at good position; (C) the post-operative CT image showed that the C2 body was ventrally slide and rotation at good position too, the spinal canal was increased significantly after operation. 

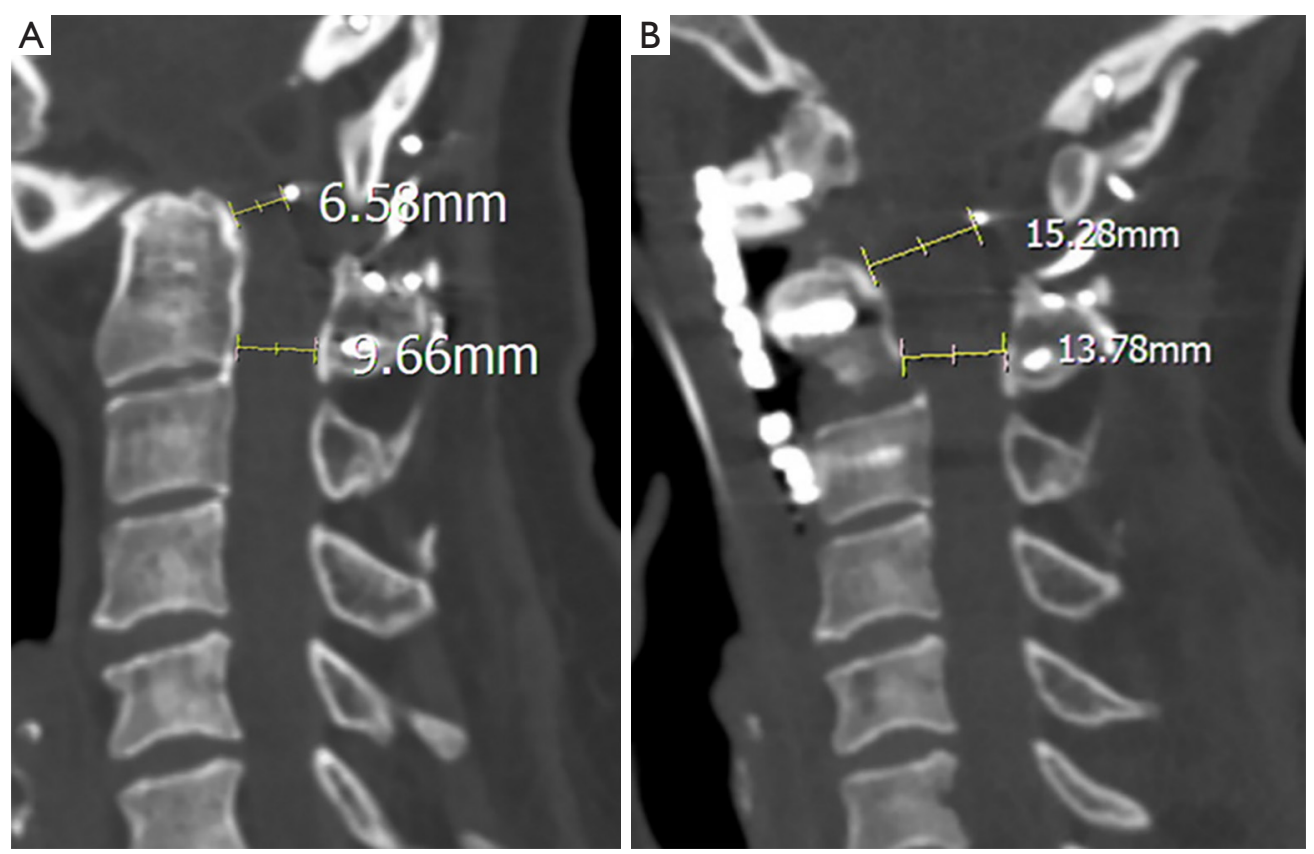

Figure 4 The changes of spinal canal between pre-operation and post-operation. (A) The pre-operative sagittal distance between the upperposterior portion of the odontoid process and transversal walking wire is $6.58 \mathrm{~mm},(\mathrm{~B})$ the postoperative image showed the significantly increased to the $15.28 \mathrm{~mm}$ after operation.

the progressive of spinal cord injury and provide the opportunity for recovery. If this patient didn't undergo posterior wire fixation and fusion at 20 years ago, a posterior $\mathrm{C} 1 / 2$ reduction and fusion (or combine posterior $\mathrm{C} 1$ arch remove) might be the first choice for her $(12,18,19)$. If the atlantoaxial dislocation is irreducible, anterior transoral atlantoaxial release or anterior submandibular retropharyngeal release will be helpful and obtain satisfied reduction and decompression $(15,20)$.

However, this patient had undertaken a posterior wire fixation and fusion 20 years ago, the pre-operative CT (current operative time) images showed that one metal wire was transversal walking through behind the spinal cord and compress the spinal cord posteriorly (Figure 1C,D). There had amount of bony fusion at posterior region of the occipital to $\mathrm{C} 4$, any operation attempt to remove this wirebone complex construction will have high risk of push the transversal walking wire anteriorly to increase the spinal cord compression, and also increase the instability of this region. Therefore, the anterior decompression will the better choice than posterior approach for this case.

Additionally, the posterior C1/2 fusion was suspected not very stable, the fused bony mass could be observed at the level of occiput to $\mathrm{C} 1$ and $\mathrm{C} 2$ to $\mathrm{C} 4$, but little between
$\mathrm{C} 1$ to $\mathrm{C} 2$ at two-dimensional CT reconstructed images. At the pre-operative ward meeting, we thought that the atlantoaxial dislocation was a gradual process in last decades, the mild fall down one month before this hospital admission like 'the last straw'. If we remove all of the odontoid process anteriorly, on the one hand, the operative procedure will also have high risk of atrogenic spinal cord injury; on another hand, the resection of odontoid process will cause excessive hemorrhage and high risk of CSF leakage, or even intracranial infection and death (21).

Therefore, we design this anterior decompression through transoral "C2 slide and rotation" technique. Of this technique, the odontoid process is not resected, but slide and rotated ventrally, therefore, the spinal canal space is restored and direct decompression is achieved, the result of this technique is similar to the anterior controllable antidisplacement and fusion in lower level of cervical spine $(22,23)$. In present case, the pre-operative sagittal distance between the upper-posterior portion of the odontoid process and transversal walking wire is $6.58 \mathrm{~mm}$ (Figure $4 A$ ), increase to the $15.28 \mathrm{~mm}$ after operation (Figure $4 B$ ). Moreover, the preserved odontoid process and C2 body served as an autogenous bone to reconstruct the anterior C1-C3 column, might have better biomechanical stability 
than the odontoid process resection technique.

To perform the operation successful, we suggest several below technique aspects should be noted. Firstly, the antero-inferior raised portion of $\mathrm{C} 2$ body and anterior raised portion of $\mathrm{C} 1$ arch should be removed by high-speed burr to prepare for the anterior cervical titanium alloy plate, as well as osteophytes if the patient have. Secondly, the internal $1 / 3$ portion of the $\mathrm{C} 1$ lateral mass also could be removed, to provide the enough space for the ventrally moved of odontoid process. Thirdly, at the procedure of bilateral osteotomy and screw insertion, the middle float C2 body should be stably held by assistant surgeon with Cook forceps or some other tools, the C2 body backward move might increase the spinal cord compression. Last but not least, we suggest that fixate the screw at the upper 1/3$1 / 2$ part of the C2 body, but not the lower 1/2 part of the C2 body, because of it could achieve a better support of the ventrally rotation of the upper portion of $\mathrm{C} 2$ body and odontoid process.

Although this technique has several advantages, we suggest that the indication could be the failure of posterior decompression or reduction, or had previous posterior surgical history that the posterior approach cannot be applied. The wire fixation and fusion technique was popularly used before the posterior screw fixation system (24-26), our present technique might be a good salvage strategy for these failed posterior fused patients. Otherwise, some irreducible atlantoaxial dislocation because of the anterior bony fusion, the anterior soft tissue release couldn't achieve reduction, our present technique could achieve anterior decompression feasibly for them in theoretically too.

Overall, we provide an anterior decompression through transoral "C2 slide and rotation" technique for salvage of failed posterior occipitocervical fusion, it could direct decompress the spinal cord anteriorly, avoid the odontoid resection, and is feasible and safe technique.

\section{Acknowledgments}

Funding: This work was supported by the National Natural Science Foundation of China (No. 81501933), Wenzhou leading talent innovative project (RX2016004), Zhejiang Provincial Medical Technology Foundation of China (2018KY129). Zhejiang Provincial Traditional Chinese Medicine Science and Technology Program (2020ZB146), Higher Education Teaching Reform Project of Wenzhou Medical University (YBJG201826).

\section{Footnote}

Conflicts of Interest: The authors have no conflicts of interest to declare.

Ethical Statement: The authors are accountable for all aspects of the work in ensuring that questions related to the accuracy or integrity of any part of the work are appropriately investigated and resolved.

\section{References}

1. Chandra PS, Kumar A, Chauhan A, et al. Distraction, compression, and extension reduction of basilar invagination and atlantoaxial dislocation: a novel pilot technique. Neurosurgery 2013;72:1040-53; discussion 1053.

2. Lall R, Patel NJ, Resnick DK. A review of complications associated with craniocervical fusion surgery. Neurosurgery 2010;67:1396-402; discussion 1402-3.

3. Wu AM, Wang XY, Chi YL, et al. Management of acute combination atlas-axis fractures with percutaneous triple anterior screw fixation in elderly patients. Orthop Traumatol Surg Res 2012;98:894-9.

4. Passias PG, Bortz CA, Segreto F, et al. Limited morbidity and possible radiographic benefit of $\mathrm{C} 2$ vs. subaxial cervical upper-most instrumented vertebrae. J Spine Surg 2019;5:236-44.

5. Sokolowski MJ, Jackson AP, Haak MH, et al. Acute outcomes of cervical spine injuries in the elderly: atlantaxial vs subaxial injuries. J Spinal Cord Med 2007;30:238-42.

6. Kamitani T, Nimura Y, Nagahiro S, et al. Catastrophic head and neck injuries in judo players in Japan from 2003 to 2010. Am J Sports Med 2013;41:1915-21.

7. Yamaguchi R, Makino Y, Inokuchi G, et al. Fatal atlantoaxial dislocation due to an odontoid synchondrosis fracture in a child with chromosome 9 abnormality: A case report. J Forensic Leg Med 2019;61:92-6.

8. Goel A, Jain S, Shah A, et al. Atlantoaxial Fixation for Odontoid Fracture: Analysis of 124 Surgically Treated Cases. World Neurosurg 2018;110:558-67.

9. Wu AM, Chi YL, Weng W, et al. Percutaneous anterior occiput-to-axis screw fixation: technique aspects and case series. Spine J 2013;13:1538-43.

10. Tang X, Tan M, Yi P, et al. Atlantoaxial dislocation and os odontoideum in two identical twins: perspectives on etiology. Eur Spine J 2018;27:259-63.

11. Guan J, Chen Z, Wu H, et al. Effectiveness of posterior reduction and fixation in atlantoaxial dislocation: a 
retrospective cohort study of 135 patients with a treatment algorithm proposal. Eur Spine J 2019;28:1053-63.

12. Shao J, Gao Y, Gao K, et al. Comparison of imaging parameters pre- and post- reductive procedure for atlantoaxial dislocation via posterior fixation using pedicle screw and rod: a cross-sectional study. BMC Musculoskelet Disord 2019;20:451.

13. Mummaneni PV, Haid RW. Transoral odontoidectomy. Neurosurgery 2005;56:1045-50; discussion 1045-50.

14. Li C, Duan J, Li L. Anterior submandibular retropharyngeal odontoid osteotomy and posterior atlantoaxial fusion for irreducible atlantoaxial dislocation associated with odontoid fracture malunion. Eur Spine J 2018;27:292-7.

15. Wang C, Yan M, Zhou HT, et al. Open reduction of irreducible atlantoaxial dislocation by transoral anterior atlantoaxial release and posterior internal fixation. Spine (Phila Pa 1976) 2006;31:E306-13.

16. Guan J, Chen Z, Wu H, et al. Is anterior release and cervical traction necessary for the treatment of irreducible atlantoaxial dislocation? A systematic review and metaanalysis. Eur Spine J 2018;27:1234-48.

17. Srivastava SK, Aggarwal RA, Nemade PS, et al. Singlestage anterior release and posterior instrumented fusion for irreducible atlantoaxial dislocation with basilar invagination. Spine J 2016;16:1-9.

18. Shao J, Gao YZ, Gao K, et al. Posterior Screw-Rod Fixation and Selective Axial Loosening for the Treatment of Atlantoaxial Instability or Dislocation Caused by Os Odontoideum: A Case Series for a Single Posterior Approach. World Neurosurg 2019;132:e193-201.

Cite this article as: $\mathrm{Wu} \mathrm{A}$, Jin H, Dou H, Pan X, Sheng S, Huang C, Wang X. Anterior decompression through transoral axis slide and rotation osteotomy for salvage of failed posterior occipitocervical fusion: a novel technique note. Ann Transl Med 2020;8(4):129. doi: 10.21037/atm.2020.02.39
19. Fung M, Frydenberg E, Barnsley L, et al. Clinical and radiological outcomes of image guided posterior $\mathrm{C} 1-\mathrm{C} 2$ fixation for atlantoaxial osteoarthritis (AAOA). J Spine Surg 2018;4:725-35.

20. Ren X, Gao F, Li S, et al. Treatment of irreducible atlantoaxial dislocation using one-stage retropharyngeal release and posterior reduction. J Orthop Surg (Hong Kong) 2019;27:2309499019870465.

21. Shriver MF, Kshettry VR, Sindwani R, et al. Transoral and transnasal odontoidectomy complications: A systematic review and meta-analysis. Clin Neurol Neurosurg 2016;148:121-9.

22. Sun J, Shi J, Xu X, et al. Anterior controllable antidisplacement and fusion surgery for the treatment of multilevel severe ossification of the posterior longitudinal ligament with myelopathy: preliminary clinical results of a novel technique. Eur Spine J 2018;27:1469-78.

23. Lee DH, Cho JH, Lee CS, et al. A novel anterior decompression technique (vertebral body sliding osteotomy) for ossification of posterior longitudinal ligament of the cervical spine. Spine J 2018;18:1099-105.

24. Winegar CD, Lawrence JP, Friel BC, et al. A systematic review of occipital cervical fusion: techniques and outcomes. J Neurosurg Spine 2010;13:5-16.

25. Griswold DM, Albright JA, Schiffman E, et al. Atlantoaxial fusion for instability. J Bone Joint Surg Am 1978;60:285-92.

26. Vender JR, Rekito AJ, Harrison SJ, et al. The evolution of posterior cervical and occipitocervical fusion and instrumentation. Neurosurg Focus 2004;16:E9. 